\title{
Study on Supply Chain Alliance Partner Selection under Low Carbon Environment
}

\author{
Yu Yang ${ }^{1}$, Zeyou Hu${ }^{1}$, Lu Gan ${ }^{1, \text { a }}$ \\ ${ }^{1}$ Sichuan Agricultural University, Chengdu 611830, China. \\ aganlu_swe_1119@sina.cn
}

\begin{abstract}
Keywords: Supply Chain Alliance, partner selection method, Fuzzy comprehensive evaluation, Low Carbon Environment, risk analysis.
\end{abstract}

\begin{abstract}
With increasing attention to low carbon environment, the research on selecting appropriate supply chain alliance partner is becoming increasingly important. This paper investigated partner selection method for setting up supply chain alliance in low carbon environment. A systematic selection method consisting of three phases was proposed. Phase one contains a preliminary selection process which was conducted by using ethical relationship theory and TOPSIS algorithm. An evaluation system based on improved fuzzy comprehensive evaluation was developed in phase two to evaluate each partner candidates. In the third phase, a qualitative analysis for overall risk of the alliance was conducted by combining risk management and fuzzy theory. The optimal supply chain alliance partner was therefore selected by considering whether the overall risk exceeds the rational range. Finally, a case study was carried out to demonstrate the feasibility and practicability of the proposed method.
\end{abstract}

\section{Introduction}

With continuous development of society, governments and enterprises are paying more and more attention to "low carbon economy" worldwide. Meanwhile, with the rapid development of economy, enterprise competition is becoming increasingly fierce and supply chain alliance with competitive advantage has increased rapidly. Study of supply chain alliance partner selection approaches is significant under low carbon environment. At present, many partner selection approaches have been proposed while each of them has its own limitations. For example, the genetic algorithm and neural network arithmetic approaches are time-consuming and labor-intensive and struggle with the issue of initial value determination; the two-phase approach, fuzzy comprehensive evaluation method and fuzzy analytic hierarchy process ${ }^{[1]}$ have difficulty in weight determination; and case reasoning and ant colony algorithm are complex and difficult to select appropriate parameters. Thus, this paper proposes a scientific and comprehensive low carbon supply chain alliance risk evaluation index system by using an improved fuzzy comprehensive evaluation method and establishes a reasonable evaluation system on the basis of supply chain alliance partners' main evaluation index ${ }^{[2]}$ and risk factors, which are of considerable significance to help enterprises in selecting low carbon supply chain alliance partner.

In this paper, a "three-phase approach" is proposed for choosing partners: The first phase screens potential partners under relational theory and completes with primary election through TOPSIS algorithm. In the second phase, the risks of the primary elected partners are calculated with genetic algorithm and the desired partners are selected on the basis of fuzzy comprehensive evaluation method. Finally, the overall risk of the supply chain alliance formed by the selected partners is measured through fuzzy analytic hierarchy process and risk decision analysis matrix. If the alliance risk value stays in a reasonable range, the alliance would be considered as competitive and acceptable. Otherwise, the alliance would be considered as risky and would be rejected.

\section{Primary Screening}

Since it is not easy for decision makers to give an accurate number (which will more often than not be replaced by a fuzzy evaluation) when evaluating the specific attribute and weight of each 
partner, in this phase, decisions were made by using the fuzzy multiple attribute decision-making method [3]. To quickly make reasonable choices among dozens of candidates, relational theory was applied for the screening of potential partners.

Filtering Stage. Relational theory provides enterprises and decision-makers with five dimensions in choosing partners, namely, time of duration, frequency of interaction, diversity of contact channels, symmetry, and promotion of cooperation.

Table 1 below illustrates the five dimensions mentioned above and their corresponding filtering criteria. Combined with business process between supply chain partners, table 1 can be further developed into a corresponding data collection table. The senior managers in chain alliance can quickly and effectively eliminate the unqualified potential partners through a combination of their own knowledge and the results from the data collection table. This stage helps in reducing the number of potential partners and the load of subsequent quantitative analysis.

Table 1 Five dimensions of relational theory ${ }^{[4]}$

\begin{tabular}{cc}
\hline Dimensions & Corresponding filtering criteria \\
\hline Duration & Lasting period of the cooperative relation \\
Frequency & Frequency of contact in unit time \\
Diversity & Number of different ways for interaction \\
Symmetry & Depth and degree of mutual integration \\
Possible promotion & Degree of knowledge sharing and willingness to cooperate \\
\hline
\end{tabular}

Primary Selection Stage. This stage is performed based on trapezoidal fuzzy number based TOPSIS method, and ranks the candidate enterprises with approximate fuzzy positive/negative ideal solution.

Determination of Evaluation Index. A supply chain consists of suppliers, manufacturers, distributors and retailers. This paper takes manufacturing enterprises as the core consideration of supply chain when considering the choice of partners. Supply chain partner selection is done through both quantitative and qualitative indicators. After checking related literature, the authors concluded that stability of product delivery, the quality of products and services, the price of products and services, the enterprise environment, the flexibility of information system, and the technical ability are the main evaluation indices.

Fuzzy Multi-Attribute Decision Making Method. Solution lies in the using of L - R trapezoid fuzzy numbers. Accurate number $\mathrm{a}=(\mathrm{a}, \mathrm{a}, \mathrm{a}, \mathrm{a})=(\mathrm{a}, \mathrm{a}, 0,0)$, interval number $(\mathrm{a}, \mathrm{b})=(\mathrm{a}, \mathrm{a}, \mathrm{b}, \mathrm{b})=$ $(\mathrm{a}, \mathrm{b}, 0,0)$, triangular fuzzy $(\mathrm{a}, \mathrm{b}, \mathrm{c})=(\mathrm{a}, \mathrm{b}, \mathrm{b}, \mathrm{c})=(-\mathrm{a}, \mathrm{b}, \mathrm{b}, \mathrm{b}, \mathrm{c}-\mathrm{b})$. Generally trapezoidal fuzzy numbers $(a, b, c, d)=(b, c, b, a, d-c)[5]$.

For a known decision matrix D:

$$
\mathrm{D}=\underset{\mathrm{A}_{n}}{A_{1}}\left[\begin{array}{ccc}
x_{11} & \cdots & x_{1 n} \\
\vdots & \ddots & \vdots \\
x_{m 1} & \cdots & x_{m n}
\end{array}\right]
$$

if $x_{i j}(\mathrm{i} \in \mathrm{M}, \mathrm{j} \in \mathrm{N})$ is an accurate number, both interval number and triangular fuzzy number can be converted to $\mathrm{L}$ - $\mathrm{R}$ trapezoid fuzzy numbers according to the above equation and be noted as $\tilde{\mathrm{x}}_{i j}=\left(a_{i j}, b_{i j} c_{i j}, d_{i j}\right)$. According to the preferences of decision makers to different attributes, the importance degree of each attribute fuzzy weight vector can be noted as $\widetilde{w}=\left(\widetilde{w}_{1}, \cdots, \widetilde{w}_{m}\right)^{T}$ in the equation, $\widetilde{\mathrm{w}}_{j}=\left(\alpha_{j}, \beta_{j}, \gamma_{j}, \delta_{j}\right)$. In multiple-attribute decision making situations, the attribute of would-be decided object can be divided into two categories - benefits and costs. The fuzzy value of the indexes and weights should be turned into accurate quantitative value for further analysis, table 2 below shows how the transformation works: 
Table 2 Meaning of fuzzy number in attributes and weights evaluation context ${ }^{[6]}$

\begin{tabular}{cccccc}
\hline Benefits & Lowest & Low & Medium & High & Highest \\
\hline Costs & Highest & High & Medium & Low & Lowest \\
Weights & Least Important & Less Important & Medium & Important & Most Important \\
Fuzzy Number & $(0,0,0,0.2)$ & $(0,0,0.1,0.3)$ & $(0.3,0.5,0.5,0.7)$ & $(0.7,0.9,1,1)$ & $(0.8,1,1,1)$ \\
\hline
\end{tabular}

Combined with TOPSIS algorithm of multiple attribute decision making, the following steps are followed to solve fuzzy multiple attribute decision making problems ${ }^{[7]}$.

Step 1: To standardize the decision matrix; each element in the decision matrix is standardized as $[0,1]$ model, using linear transformation:

$$
r_{i j}=\left\{\begin{array}{l}
\frac{x_{i j}}{x_{j}}, \forall \mathrm{j} \in \mathrm{N}, f_{j} \text { Benefit valuing type } \\
\frac{\tilde{x}_{j}^{-}}{x_{i j}}, \forall \mathrm{j} \in \mathrm{N}, f_{j} \text { Cost valuing type }
\end{array}\right.
$$

xij and its corresponding $r_{i j}$ are fuzzy numbers, $\tilde{x}_{j}^{+}$and $\tilde{\mathrm{x}}_{j}^{-}$can be noted as $\tilde{x}_{j}^{+}=$ $\left(a_{j}^{+}, b_{j}^{+}, c_{j}^{+}, d_{j}^{+}\right), \tilde{\mathrm{x}}_{j}^{-}=\left(a_{j}^{-}, b_{j}^{-}, c_{j}^{-}, d_{j}^{-}\right)$. Thus equation (1) is converted to:

$$
\tilde{r}_{i j}=\left\{\begin{array}{l}
\frac{\tilde{x}_{i j}}{\tilde{x}_{j}^{+}}=\left(\frac{a_{i j}}{d_{j}^{+}}, \frac{b_{i j}}{c_{j}^{i}}, \frac{c_{i j}}{b_{j}^{+}}, \frac{d_{i j}}{a_{j}^{i}}\right) \text { Benefit valuing type } \\
\frac{\tilde{x}_{j}^{-}}{\tilde{x}_{i j}}=\left(\frac{a_{j}^{-}}{a_{i j}}, \frac{b_{j}^{-}}{c_{i j}}, \frac{c_{j}^{-}}{b_{i j}}, \frac{d_{j}^{-}}{a_{i j}}\right) \text { Cost valuing type }
\end{array}\right.
$$

Step 2: To construct weighting to normalize decision matrix $V$ as follows:

$$
\mathrm{V}_{i j}=r_{i j} w_{j}, \quad \forall \mathrm{i} \in \mathrm{M} ; j \in N \text {. (3) }
$$

Because $\mathrm{r}_{i j}$ and $w_{j}$ are fuzzy numbers, equation 3 , which is also fuzzy, can be transformed into:

$$
\widetilde{\mathrm{V}}_{i j}=\left\{\begin{array}{l}
\tilde{r}_{i j} \times \widetilde{w}_{j}=\left(\frac{a_{i j}}{d_{j}^{+}} \alpha_{j}, \frac{b_{i j}}{c_{j}^{+}} \beta_{j}, \frac{c_{i j}}{b_{j}^{+}} \gamma_{j}, \frac{d_{i j}}{a_{j}^{+}} \delta_{j}\right), \text { Benefit attributes } \\
\tilde{r}_{i j} \times \widetilde{w}_{j}=\left(\frac{a_{j}^{-}}{d_{i j}} \alpha_{j}, \frac{b_{j}^{-}}{c_{i j}} \beta_{j}, \frac{c_{j}^{-}}{b_{i j}} \gamma_{j}, \frac{d_{j}^{-}}{a_{i j}} \delta_{j}\right), \quad \text { Cost attributes }
\end{array}\right.
$$

from equation $\mathrm{V}=\left(\tilde{\mathrm{v}}_{i j}\right) \mathrm{m} \times \mathrm{n}$.

Step 3: To determine the positive ideal solution $\tilde{\mathrm{x}}^{+}$and negative ideal solution $\tilde{x}^{-}$, respectively noted as:

$$
\tilde{x}^{+}=\left\{\tilde{v}_{1}^{+}, \tilde{v}_{2}^{+}, \cdots, \tilde{v}_{n}^{+}\right\}, \quad \tilde{x}^{-}=\left\{\tilde{v}_{1}^{-}, \tilde{v}_{2}^{-}, \cdots \tilde{v}_{n}^{-}\right\} .
$$

In this equation, $\tilde{\mathrm{v}}_{j}^{+}=\max _{i} \tilde{v}_{i j}, \tilde{v}_{j}^{-}=\min _{i} \tilde{v}_{i j}$.

Step 4: To calculate distance $s_{i}^{+}$and $s_{i}^{-}$.[8]

The distance of the positive ideal solution in each scheme is noted as:

$$
\mathrm{S}_{i}^{+}=\sqrt{\sum_{j=1}^{n}\left(\tilde{v}_{i j}-\tilde{v}_{j}^{+}\right)}, \quad \forall \mathrm{i} \in \mathrm{M} \text {. (6) }
$$

The distance of the positive ideal solution in each scheme is noted as:

$$
\mathrm{S}_{i}^{-}=\sqrt{\sum_{j=1}^{n}\left(\tilde{V}_{i j}-\tilde{v}_{j}^{+}\right)}, \quad \forall \mathrm{i} \in \mathrm{M} . \quad \text { (7) }
$$

Step 5: To calculate the relative close degree index $C_{i}$ of each scheme and understanding

$$
C_{i}=S_{i}^{-} /\left(S_{i}^{-}+S_{i}^{+}\right), \quad \forall \mathrm{i} \in \mathrm{M} \text {. (8) }
$$

So can the solutions be sorted according to the size of $C_{\mathrm{i}}$. According to the specific requirements of low carbon supply chain alliance, the choices are sorted out from big to small in number and make it liable to select "a” number of suppliers, "b" number of distributors, and "c" number of 
retailers.

\section{Partners Selection}

To make a second and subtler selection among the suppliers (amount a), distributors (amount b), and retailers (amount c) which are selected after primary screening, genetic algorithm is used to determine the weights of evaluation indexes of low carbon supply chain alliance partners. Furthermore, a combination with fuzzy comprehensive evaluation method is performed to select the optimal partner.

Evaluate Partners. To evaluate the partners left after primary selection, first determine the chosen object set $X=\left\{x_{1}, X_{2}, X_{3} \ldots x_{n}\right\}$, here object set refers to the collection of candidates(partners) qualified for second selection. Secondly, determine factor sets of the candidates. According to the analysis of low carbon supply chain alliance, we get the partner evaluation index system ${ }^{[2]}$ in table 3 , it can be seen at this point that there is a need for multi-level comprehensive evaluation. So factors set can be divided into primary factors set $U=\left\{\mathrm{u}_{1}, \mathrm{u}_{2}, \mathrm{u}_{3} \ldots \mathrm{UN}\right\}$, and secondary factors set $\mathrm{U}_{\mathrm{i}}=\left\{\mathrm{u}_{\mathrm{i} 1}, \mathrm{u}_{\mathrm{i} 2}, \mathrm{u}_{\mathrm{i} 3} \ldots \mathrm{u}_{\mathrm{in}}\right\}$.

Then establish comments set of evaluation objects $V=\left\{\mathrm{v}_{1}, \mathrm{v}_{2} \ldots \mathrm{v}_{\mathrm{n}}\right\}$, the five levels in comments set are: excellent, good, average, poor, and fairly poor)

Finally, through the single factor evaluation, we can determine the fuzzy values of factor set V in the mapping $R_{i}=\left(r_{i 1} r_{i 2} \ldots\right.$, rim), namely $f: U \rightarrow V$. And acquire the single factor evaluation matrix $R$ $=\left(\mathrm{r}_{\mathrm{ij}}\right)_{\mathrm{n} \times \mathrm{m}}$.

Table 3 Low carbon supply chain alliance partner evaluation index system

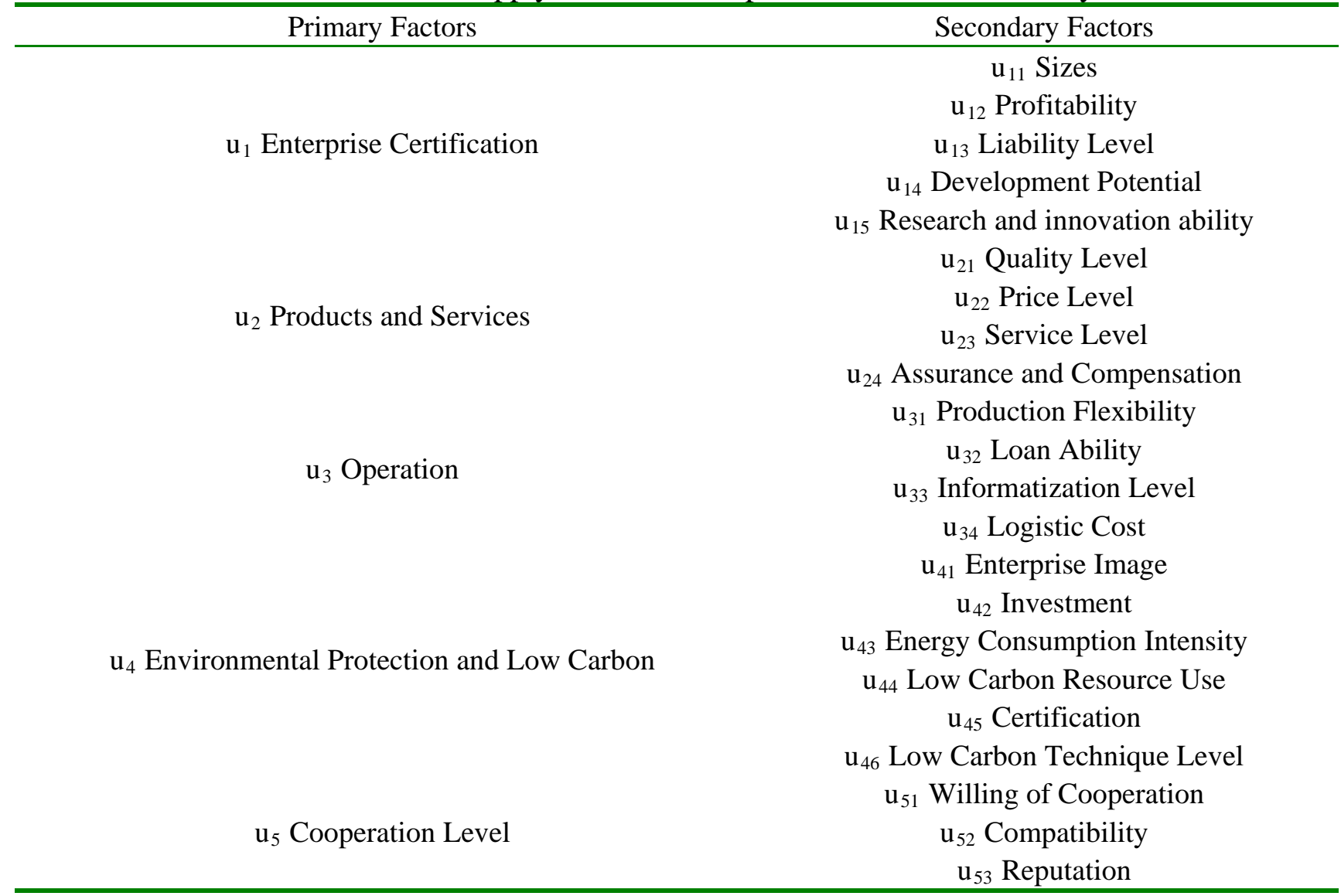

Weights Determination. When applying genetic algorithm to determine weights, this paper adopts MATLAB genetic algorithm toolbox (GAOT), and it is done through the GUI interface. First, fitness function is determined ${ }^{[9]}$. Assume an individual $a_{i}$, its corresponding weights allocation is $u_{i 1}, u_{i 2}, \ldots$. The comprehensive decision noted as $b^{\prime}$, b stands for given comprehensive decision. Thus, the fitness definition of $a_{i}$ is: 


$$
\mathrm{f}\left(a_{i}\right)=\frac{\sum_{i}^{m}\left(b^{\prime}(k) \wedge b(k)\right)}{\sum_{i}^{m}\left(b^{\prime}(k) \vee \mathrm{b}(\mathrm{k})\right)}
$$

$\Lambda 、 \vee$ respectively stands for taking maximum and taking minimum, $b(k)$ represents the $k$ th component of b', accordingly $\mathrm{b}(\mathrm{k})$ represents the $k$ th component of $\mathrm{b}$. As the genetic algorithm toolbox is always trying to find the minimum value of fitness function, to get maximum of the objective function $f\left(a_{i}\right)$, we can turn the other way round to calculate the minimum value of function $\mathrm{g}\left(\mathrm{a}_{i}\right)=-\mathrm{f}\left(a_{i}\right)$ and compose a $\mathrm{M}$ file of fitness function according to the above formula.

Then, we input the relevant parameters in interface, using genetic algorithm tool (GUI). Hereafter click "Start" button to run genetic algorithm (ga), the running results will appear on "Status and results" pane, and we got the corresponding weights $\mathrm{W}=\left(w_{1}, w_{2}, \ldots, w_{i}\right)$, and $\sum_{i=1}^{n} w_{i}=1$.

Matrix R Establishment. Carry out fuzzy evaluation of single factor, establish fuzzy relationship matrix $\mathrm{R}=\left(r_{i j}\right)_{n \times m}$

$$
\mathrm{R}=\left[\begin{array}{ccc}
r_{11} & \cdots & r_{1 n} \\
\vdots & \ddots & \vdots \\
r_{m i} & \cdots & r_{m n}
\end{array}\right]
$$

Comprehensive Evaluations. Multiply single factor evaluation matrix of $\mathrm{N}$ factors $\mathrm{R}=\left(r_{i j}\right)_{n \times m}$ and weight $\mathrm{W}=\left(w_{1}, w_{2}, \ldots, w_{i}\right)$, get the comprehensive evaluation result $\mathrm{B}$. The optimal results come out according to the maximum membership degree principle.

\section{Risk Assessment}

The Risk of Low Carbon Supply Chain Alliance. Low carbon supply chain alliance risk management concerns the identification of risk factors, the assessment of risk properties, the calculation of risk occurrence probability and risk severity ${ }^{[10]}$, with a final determination of whether the overall risk value is kept within an acceptable range. First of all, we analyzed the risk factors within a low carbon supply chain alliance. Low carbon supply chain alliance differs from traditional supply chain mainly in the following aspects: (1) Limited/restricted carbon emission rights in production; (2) Carbon emissions rights can be regarded as item for trade, or, tradable resources; (3) Economic entity providing low carbon related services appear in the new economic system, such as carbon emissions exchange that provides enterprises with energy-saving and emission-reducing technology, carbon emissions trading platform, etc.; (4) The structural change of traditional supply chain alliance, in which new economic entities appear. And members of the supply chain trade carbon emission rights with the help of carbon trading related enterprises; (5) The consumer's increasing awareness ${ }^{[11]}$ of carbon reduction when considering consumer value. Table 4 shows the risks of low carbon supply chain alliance.

Risk Assessment of Low Carbon Supply Chain Alliance. Two major aspects must be sufficiently considered in the risk assessment of supply chain alliance: first, the probability of risks; second, the severity of possible risks. After the establishment of low carbon supply chain alliance, to determine whether the overall risk is kept within acceptable range, we use the risk decision analysis matrix to make overall risk assessment of the alliance. Risk decision matrix is a risk decision analysis based on risk probability and the corresponding risk severity ${ }^{[12]}$. The importance of each risk factor can be calculated by the following equation:

$$
\text { Risk = risk probability } \times \text { risk severity }
$$

To Determine Value at Risk. In the initial data collection period, we use the questionnaire survey method. Experts vote their opinion on five options (very unlikely, unlikely, even, likely, very likely), (very low, low, medium, significant, catastrophic) to suggest the risk probability and risk severity. In order to get the risk value of each factor, we use the fuzzy value to represent the risk value. The fuzzy value table (Table 5) originates from the result of PMBOK (2004 edition) and 
shows the fuzzy value of risk. Table 5 shows the risk probability and the risk severity function ${ }^{[13]}$. Table 4 Risk factors of low carbon supply chain alliance

\begin{tabular}{|c|c|c|c|c|c|c|}
\hline Supply Risk & \multicolumn{2}{|c|}{ Demand Risk } & \multicolumn{3}{|c|}{ Manufacture Risk } & Information Risk \\
\hline Relation with Suppliers & $\begin{array}{r}\text { Customer Mana } \\
\text { Ability }\end{array}$ & ment & \multicolumn{3}{|c|}{ Productivity } & $\begin{array}{l}\text { IT and Software } \\
\text { System }\end{array}$ \\
\hline $\begin{array}{l}\text { Deliverability of } \\
\text { Suppliers }\end{array}$ & Change of Cus & mers & \multicolumn{3}{|c|}{ Product and Process Design } & $\begin{array}{l}\text { Information Twist } \\
\text { and Distortion }\end{array}$ \\
\hline Supplier Deliveries & $\begin{array}{r}\text { Market Dem } \\
\text { Fluctuatio }\end{array}$ & & \multicolumn{3}{|c|}{ Inventory Control } & $\begin{array}{l}\text { Integrity of } \\
\text { Information }\end{array}$ \\
\hline $\begin{array}{l}\text { Suppliers Network } \\
\text { Design }\end{array}$ & $\begin{array}{r}\text { Customer Fin } \\
\text { Situation }\end{array}$ & & \multicolumn{3}{|c|}{ Quality Control } & \\
\hline $\begin{array}{l}\text { Suppliers Operation } \\
\text { Conditions }\end{array}$ & \multirow{2}{*}{\multicolumn{2}{|c|}{$\begin{array}{l}\text { Products and Services } \\
\text { Customer Low Carbon } \\
\text { Requirements }\end{array}$}} & \multicolumn{3}{|c|}{$\begin{array}{c}\text { Carbon Allowance Exhausted } \\
\text { and Purchase Delay }\end{array}$} & \\
\hline Natural Disaster & & & & & & \\
\hline \multicolumn{7}{|l|}{$\begin{array}{c}\text { Carbon Emission Policy } \\
\text { Adjustments }\end{array}$} \\
\hline \multicolumn{7}{|c|}{ Table 5 Risk decision analysis matrix } \\
\hline Severity Probability & Very low & lo & & Medium & High & Catastrophic \\
\hline Very Unlikely & Low & $\mathrm{Lc}$ & & Medium & Significant & Significant \\
\hline Unlikely & Low & $\mathrm{Lc}$ & & Medium & Significant & High \\
\hline Even & Low & Mec & & Significant & High & High \\
\hline Likely & Medium & Signi & ant & Significant & High & High \\
\hline Very Likely & Significant & Signi & ant & High & High & High \\
\hline
\end{tabular}

By using this method, the probability of the data and the severity of each factor are gathered in the form of linguistic data. After transforming these evaluation languages into triangular fuzzy numbers, we get the fuzzy values of the probability and severity of each linguistic variable shown in table 6.

Table 6 Linguistic values and fuzzy values of the probability, severity, and risk

\begin{tabular}{cc}
\hline Linguistic Value & Fuzzy Value \\
\hline Probability of Risk Occurrence & $(0,0.125,0.25)$ \\
Very Unlikely & $(0.05,0.275,0.5)$ \\
Unlikely & $(0.25,0.5,0.75)$ \\
Even & $(0.5,0.725,0.95)$ \\
Likely & $(0.75,0.875,1)$ \\
Very Likely & \\
Severity of Risk Occurrence & $(0,1,25,2.5)$ \\
Very Little & $(0.5,2.75,5)$ \\
Little & $(2.5,5,7.5)$ \\
Medium & $(5,7.25,9.5)$ \\
High & $(7.5,8.75,10)$ \\
Catastrophic & \\
Risk value & \\
Low & $(0,0.165,0.33)$ \\
Medium & $(0.05,0.355,0.66)$ \\
Significant & $(0.33,0.64,0.95)$ \\
High & $(0.66,0.83,1)$ \\
\hline
\end{tabular}

After transforming the language variables into triangular fuzzy numbers, we assume $\tilde{A}=\left(a_{l}, a_{m}, a_{u}\right)$ as a triangular fuzzy function. For the different options of "q" number of experts, 
we use simple weighting method to collect all the preference of decision makers, the calculating formula is ${ }^{[14]}$ :

$$
\tilde{A}_{i j}=\frac{1}{q}\left(\tilde{a}_{i j}^{(1)}+\tilde{a}_{i j}^{(2)}+\cdots \tilde{a}_{i j}^{(q)}\right)=\left(\frac{\sum_{k=1}^{q} l_{i j}^{(k)}}{q}, \frac{\sum_{k=1}^{q} m_{i j}^{(k)}}{q}, \frac{\sum_{k=1}^{q} u_{i j}^{(k)}}{q}\right)
$$

Then we adopt center area method (COA) ${ }^{[15]}$ to de-fuzzy triangular fuzzy number to get the corresponding best non-fuzzy performance (BNP). In the BNP approach, its certainty value can be calculated through equation (10):

$$
\mathrm{A}=\frac{\left(a_{u}-a_{l}\right)+\left(a_{m}-a_{l}\right)}{3}+a_{l}(10)
$$

To Determine Index Weight. Construct a judgment matrix focusing on pairwise comparison. In this paper, we adopt $0.1 \sim 0.9$ five scale method, shown in table 7 . Get fuzzy complementary matrix: $\mathrm{S}=\left(s_{i j}\right)_{n \times n}{ }^{[16]}$.

Table 7 Criteria for significance judgment ${ }^{[17]}$

\begin{tabular}{cc}
\hline Scales & Implication \\
\hline 0.1 & element B far more important than element A \\
0.3 & element B more important than element A \\
0.5 & element B equally important with element A \\
0.7 & element A more important than element B \\
0.9 & element A far more important than element B \\
\hline
\end{tabular}

Notes: $0.2,0.4,0.6,0.8$ can take adjacent value in five scales.

Matrix A sum in row:

Mathematical conversion:

$$
r_{i}=\sum_{k=1}^{n} s_{i k}(i=1,2, \ldots, n)
$$

$$
r_{i j}=\left[\frac{\left(r_{i}-r_{j}\right)}{2(n-1)}\right]+0.5(12)
$$

Fuzzy consistent matrix acquired: $\mathrm{R}=\left(\mathrm{r}_{i j}\right)_{n * n}$, ordering weight acquired: $\mathrm{w}=\left(w_{1}, w_{2}, \ldots w_{n}\right)$ satisfies:

$$
\boldsymbol{w}_{\boldsymbol{i}}=\left[\sum_{k=1}^{n} s_{i j}+\left(\frac{n}{2}\right)-1\right] /[n(n-1)](i=1,2, \ldots, n)(13)
$$

To Determine the Overall Risk. The overall risk of the alliance $Q$ is calculated by multiplying the risk of each factor $A_{i j}$ with their corresponding weight $W_{i}$, as shown in equation below:

$$
Q=\sum_{k=1}^{n} w_{i} \times A_{i j}(14) \text {. }
$$

Finally, we define $Q$ ranging from 0 to 0.75 as the desired threshold range, which means any $Q$ exceeds 0.75 would be considered as high risk alliance that should not be formed and need to reselect the partners.

\section{Case Analysis}

To choose supply chain alliance partners under low carbon environment, a mobile phone manufacturer makes respective selections in its suppliers, distributors and retailers.

Step 1. After filtering unqualified partners according relational theory, there are 7 suppliers, 6 distributors, and 6 retailers left. To establish three good cooperative relationships, an expert group is nominated to analyze every index and the index weight of each potential partner and determine the corresponding fuzzy number. Afterwards the final ranking of candidates will be given through approximate fuzzy positive/negative ideal solution based on trapezoidal fuzzy number TOPSIS method.

Then use MATLAB software to deal with the data obtained, and get indicator $C_{i}$, which shows the closeness between the seven candidate suppliers named $A_{1}, A_{2}, A_{3}, A_{4}, A_{5}, A_{6}, A_{7}$ and the ideal solutions are shown in table 8 . Based on the results, $A_{3}, A_{6}, A_{4}$ are selected. Similarly, the results of 
candidate distributors and retailers are shown in table 9 and table 10, respective. Accordingly, the selections for distributors and retailers are $\mathrm{B}_{6}, \mathrm{~B}_{3}, \mathrm{~B}_{2}$ and $\mathrm{D}_{1}, \mathrm{D}_{6}, \mathrm{D}_{4}$, respectively.

Table 8 Closeness results for candidate suppliers

\begin{tabular}{cccccccc}
\hline Suppliers & $\mathrm{A}_{1}$ & $\mathrm{~A}_{2}$ & $\mathrm{~A}_{3}$ & $\mathrm{~A}_{4}$ & $\mathrm{~A}_{5}$ & $\mathrm{~A}_{6}$ & $\mathrm{~A}_{7}$ \\
\hline Closeness & 0.4094 & 0.3496 & 0.6793 & 0.6395 & 0.5452 & 0.6458 & 0.5271 \\
\hline \multicolumn{7}{c}{ Table } & Closeness results for candidate distributors \\
\hline Distributors & $\mathrm{B}_{1}$ & $\mathrm{~B}_{2}$ & $\mathrm{~B}_{3}$ & $\mathrm{~B}_{4}$ & $\mathrm{~B}_{5}$ & $\mathrm{~B}_{6}$ \\
\hline Closeness & 0.4688 & 0.5709 & 0.6932 & 0.5446 & 0.3143 & 0.7073 \\
\hline \multicolumn{7}{c}{ Table } & 10 Closeness results for candidate retailers \\
\hline Retailers & $\mathrm{D}_{1}$ & $\mathrm{D}_{2}$ & $\mathrm{D}_{3}$ & $\mathrm{D}_{4}$ & $\mathrm{D}_{5}$ & $\mathrm{D}_{6}$ \\
\hline Closeness & 0.7085 & 0.4364 & 0.4601 & 0.4979 & 0.4554 & 0.6944 \\
\hline
\end{tabular}

Step 2. We invite 10 experts to rate the selected enterprises. After optimizing through genetic algorithm the factor weights given by experts, we get factors' weights at all levels shown in Table 11.

Table 11 Factors' weights at all levels

\begin{tabular}{ccccccccccc}
\hline $\begin{array}{c}\text { Primary } \\
\text { factors }\end{array}$ & $\begin{array}{c}\text { Secondary } \\
\text { factors }\end{array}$ & $\mathrm{A}_{3}$ & $\mathrm{~A}_{6}$ & $\mathrm{~A}_{4}$ & $\mathrm{~B}_{6}$ & $\mathrm{~B}_{3}$ & $\mathrm{~B}_{2}$ & $\mathrm{D}_{1}$ & $\mathrm{D}_{6}$ & $\mathrm{D}_{4}$ \\
\hline \multirow{6}{*}{0.2011} & 0.1274 & 0.78 & 0.92 & 0.88 & 0.9 & 0.8 & 0.9 & 0.88 & 0.86 & 0.82 \\
& 0.3983 & 0.74 & 0.86 & 0.8 & 0.84 & 0.82 & 0.86 & 0.84 & 0.82 & 0.8 \\
& 0.1637 & 0.86 & 0.82 & 0.78 & 0.76 & 0.82 & 0.68 & 0.74 & 0.7 & 0.78 \\
& 0.1316 & 0.84 & 0.82 & 0.78 & 0.92 & 0.88 & 0.72 & 0.8 & 0.76 & 0.9 \\
0.2309 & 0.2690 & 0.9 & 0.86 & 0.82 & 0.72 & 0.68 & 0.7 & 0.7 & 0.62 & 0.8 \\
\hline \multirow{5}{*}{0.2402} & 0.2988 & 0.74 & 0.78 & 0.76 & 0.74 & 0.76 & 0.74 & 0.88 & 0.8 & 0.72 \\
& 0.2513 & 0.88 & 0.76 & 0.76 & 0.74 & 0.7 & 0.8 & 0.86 & 0.8 & 0.84 \\
& 0.2515 & 0.76 & 0.84 & 0.7 & 0.76 & 0.74 & 0.78 & 0.88 & 0.82 & 0.72 \\
& 0.1984 & 0.76 & 0.68 & 0.76 & 0.82 & 0.64 & 0.68 & 0.78 & 0.78 & 0.82 \\
\hline & 0.2698 & 0.78 & 0.72 & 0.78 & 0.74 & 0.78 & 0.9 & 0.88 & 0.86 & 0.68 \\
& 0.2295 & 0.76 & 0.84 & 0.78 & 0.78 & 0.68 & 0.8 & 0.92 & 0.78 & 0.66 \\
0.1495 & 0.2178 & 0.86 & 0.84 & 0.9 & 0.8 & 0.74 & 0.84 & 0.76 & 0.74 & 0.84 \\
& 0.0965 & 0.8 & 0.76 & 0.64 & 0.66 & 0.78 & 0.76 & 0.88 & 0.84 & 0.74 \\
\hline & 0.1597 & 0.62 & 0.66 & 0.62 & 0.66 & 0.68 & 0.7 & 0.6 & 0.78 & 0.78 \\
& 0.2010 & 0.72 & 0.74 & 0.72 & 0.76 & 0.66 & 0.68 & 0.72 & 0.84 & 0.66 \\
& 0.2011 & 0.66 & 0.7 & 0.68 & 0.64 & 0.88 & 0.76 & 0.68 & 0.76 & 0.68 \\
0.1783 & 0.1421 & 0.56 & 0.74 & 0.66 & 0.62 & 0.78 & 0.78 & 0.7 & 0.78 & 0.74 \\
& 0.1992 & 0.72 & 0.76 & 0.72 & 0.66 & 0.82 & 0.88 & 0.7 & 0.82 & 0.82 \\
\hline
\end{tabular}

Calculate degree of membership and get Table 12 according to Table 11 and the scores of given by experts.

Table 12 Degree of membership

\begin{tabular}{cccccccccc}
\hline Candidates & $\mathrm{A}_{3}$ & $\mathrm{~A}_{6}$ & $\mathrm{~A}_{4}$ & $\mathrm{~B}_{6}$ & $\mathrm{~B}_{3}$ & $\mathrm{~B}_{2}$ & $\mathrm{D}_{1}$ & $\mathrm{D}_{6}$ & $\mathrm{D}_{4}$ \\
\hline $\mathrm{B}_{\mathrm{i}}$ & 0.7744 & 0.7964 & 0.7741 & 0.7498 & 0.7658 & 0.789 & 0.8092 & 0.7972 & 0.7135 \\
\hline
\end{tabular}

In this case, supplier $\mathrm{A}_{6}$, distributor $\mathrm{B}_{2}$ and retailer $\mathrm{D}_{1}$ were chosen according to the maximum degree of membership principle.

Step 3. To analyze the risk of supply chain alliance through fuzzy analytic hierarchy process, determine the weight of risk factors, get table 13 . 
Table 13 Risk factors' weights

\begin{tabular}{llll}
\hline 0.275 & 0.308 & 0.217 & 0.200 \\
\hline 0.148 & 0.137 & 0.180 & 0.293 \\
0.143 & 0.170 & 0.245 & 0.317 \\
0.107 & 0.183 & 0.150 & 0.400 \\
0.126 & 0.200 & 0.230 & \\
0.164 & 0.163 & 0.195 & \\
0.181 & 0.147 & & \\
\hline
\end{tabular}

Invite experts to assess the probability and severity of the risk factors, get Table 14 .

Table 14 Assessment results of risk probability and severity

\begin{tabular}{cccc}
\hline Supply Risk & Demand Risk & Manufacture Risk & Information Risk \\
\hline Low & Low & Medium & Low \\
Fairly High & Fairly High & Fairly High & Fairly High \\
Medium & Fairly High & Fairly Low & High \\
Low & Fairly High & Fairly High & \\
Medium & Fairly High & Medium & \\
Fairly High & Low & & \\
Medium & & & \\
\hline
\end{tabular}

Solve $\mathrm{Q}=0.74<0.75$ according to the above-mentioned Table 6 and formula (14), so risk value of the alliance is kept acceptable range, and thus can be established.

\section{Conclusion}

To overcome the disadvantages of existing approaches for partner selection in low carbon supply chain appliance, this paper puts forward a three-phase approach. Phase one contains a preliminary selection process which is conducted by using ethical relationship theory and TOPSIS algorithm. Phase two involves an evaluation system based on improved fuzzy comprehensive evaluation to select candidate partners. In the third phase, the paper focuses on the analysis of risk factors in supply chain alliance and an overall risk assessment is correspondingly made. During the whole process, key factors including low carbon context and risk assessment are considered with systematic, theoretic and practical concerns. In future application, enterprises can determine the specific evaluation indicators and risk factors according to the particular needs of certain supply chain alliance.

\section{References}

[1] Yangxu Li, Application of improved fuzzy comprehensive evaluation in partner-selection in dynamic alliance, Chinese Journal of Management Science. 14 (2006) 627-631.

[2] Zhigang Ding, Qi Xu, The evaluation and selection of low carbon supply chain strategic partners, China Business and Market. 27 (2013) 102-106.

[3] Fang Wang, Qizong Wu, Fuzzy multiple attribute decision making under incomplete attribute weights based on cooperation partner of supply chain, Mathematics in Practice and Theory. 38 (2008) 18-23.

[4] Xiaojing Liu, The electronic commerce environment research of virtual enterprise partner selection. Beijing Jiaotong University. 2007.

[5] Peng Zhansheng. Fuzzy multiple attribute decision making for Selecting Cooperative R\&D Partners, Science and Technology Management Research. 27 (2007) 149-151. 
[6] Rongjun Li, Fuzzy multi-criteria decision making theory and application, Science Press, Beijing, 2002, pp. 138-198.

[7] Jiuping $\mathrm{Xu}$, Wei $\mathrm{Wu}$, Multiple attribute decision making theory and methods, Tsinghua University Press, Beijing, 2006, pp. 271-273.

[8] Zhengcheng Wang, Jinghong Tian, Liangpeng Ye, Research on selection of partner enterprises in agile supply chains, Machinery. 41 (2003) 43-45.

[9] Haijun Wang, Yu Deng, Wenhao Li, Ci Song, Application of genetic algorithm in the determination of land rating element proportion, Geomatics and Information Science of Wuhan University. 35 (2010) 240-243.

[10] Yanru Zhang, Jingxiao Chen, Quan Zheng, Fuzzy assessment method research of supply chain uncertain risk based on predilection, Operations Research and Management Science. 17 (2008) 69-73.

[11]Rongjuan Liu, Daozhi Zhao, Risk source identification and control strategy of low carbon supply chain, Journal of Wuhan University of Technology (Information \& Management Engineering). 4 (2014) 513-516.

[12] Ghaffari M, Sheikhahmadi F, Safakish G, Modeling and risk analysis of virtual project team through project life cycle with fuzzy approach, Computers \& Industrial Engineering. 72 (2014) 98-105.

[13] Ebrat M, Ghodsi R. Risk assessment of construction projects using network based adaptive fuzzy system, International Journal of Academic Research. 3 (2011) 411.

[14] Yanping Jiang, A ranking method for reciprocal judgement matrix with triangular fuzzy numbers, Systems Engineering and Electronics. 24 (2002) 34-36.

[15] Zhao R, Govind R, Algebraic characteristics of extended fuzzy numbers, Information Sciences. 54 (1991) 103-130.

[16] Jingwei Hui, The fuzzy analytic hierarchy process to choose the appropriate project management mode, Industrial Technology \& Economy. 23(2004) 73-75.

[17] Shipin Zhu, Xiaofeng Zhang, The application of investigation and expert marking method in risk assessment, Shanxi Architecture. 32 (2006) 274-275. 